\title{
El pasado de la medicina: la historia y el oficio. Entrevista con Roy Porter
}

\section{Medicine in the past: the bistory and the historian's profession. Interview with Roy Porter}

\author{
Marcos Cueto \\ Universidad Peruana Cayetano Heredia \\ Roca Bologna 633 \\ Lima 18 Perú \\ mcueto@upch.edu.pe
}

El 3 de marzo del 2002, pocos meses después que se realizó esta entrevista en Lima, murió Roy Porter, en la ciudad de Londres. Este extraordinario historiador de la medicina, la ciencia y la ilustración, encarnaba y trascendía el notable desarrollo de la historia social y de la historia de la medicina experimentado en su país en los últimos años. Las obras de Porter contienen contribuciones valiosísimas como la perspectiva de los pacientes, la interacción entre las imágenes del cuerpo y de la sociedad y la historia de la salud mental. En su país fue muy conocido no sólo por sus varias publicaciones sino por su destacada participación en programas de radio y televisión. Hace pocos años elaboró una visión panorámica de la medicina desde sus orígenes hasta el presente titulada The greatest benefit of mankind, a medical history of humanity (1997) que se há convertido en un bestseller internacional. Fue profesor del Wellcome Trust Centre for the History of Medicine de la Universidad de Londres donde sus colegas y alumnos lo recuerdan como un investigador y profesor totalmente dedicado a su trabajo. Recuerdo claramente la profundidad, la claridad, y la elegancia de sus presentaciones en Lima en agosto del año 2001; en medio de las cuales se realizo esta entrevista. Entre los planes con los que tuve el privilegio de conversar con Roy Porter, después de su paso por Lima, estuvo el de su visita a otras ciudades de América Latina como Rio de Janeiro (donde los amigos de la Casa de Oswaldo Cruz ya tenían prevista una serie de conferencias para comienzos del año 2003). La siguiente entrevista, que trata sobre aspectos esenciales de la historia de la medicina y del oficio de historiador de la medicina, es también un homenaje, al historiador notable, al ser humano excepcional.

PALAVRAS-CHAVE: Roy Porter, medicina, historia de la medicina, historiadores de la medicina.

On March 3, 2002, a few months after this interview was held in Lima, Roy Porter died in London. He was an extraordinary historian of medicine, science and the Enlightenment, 
and he both personified and transcended the remarkable development of social history and the history of medicine in his country in recent years. Porter's work included very valuable contributions to this history, such as the patients' perspective, interaction between images of the body and of society and the history of mental health. He was well known in Great Britain not only for his publications but his participation in radio and television programs. A few years ago he produced a panoramic view of medicine from its origins to the present, entitled The greatest benefit of mankind, a medical history of humanity (1997), which became an international bestseller. He was a professor at the Wellcome Trust Centre for the History of Medicine of the University of London, where his colleagues and students recall him as a researcher and teacher completely dedicated to his work. I remember clearly his profound, clear and elegant presentations in Lima in August of 2001; it in the midst of that series of talks that this interview took place. Among the plans I had the privilege to discuss with Roy Porter was, after Lima, to visit other Latin American cities; in Rio de Janeiro, his friends at the Casa de Oswaldo Cruz had scheduled a lecture series for the beginning of 2003. The interview that follows, on fundamental issues in the history of medicine and the medical historian's profession, is also an homage to this remarkable historian and exceptional human being.

KEYWORDS: Roy Porter, medicine, history of medicine, historians of medicine.

“... la enfermedad y la muerte han sido dos de los mayores determinantes de la experiencia humana".
¿Cómo se interesó en la historia de la medicina?

Fue producto de una serie de accidentes en los que hubo cierta lógica. Siempre estuve interesado en la historia y cuando empecé a especializarme me orienté a la historia de la ciencia, especialmente a la historia de la geología. Encontré que muchos de los primeros científicos eran médicos y ello despertó mi interés en el papel de los doctores en la sociedad. Posteriormente eso me llevó a estudiar el desarrollo de la medicina. Asimismo, el haber sido entrenado como un historiador social significó que me interesase más en las relaciones sociales de la medicina — es decir la relación entre médicos y pacientes — que en la medicina en sí misma. Así que fue un interés general en la historia lo que me llevó a la historia de la medicina.

Sus libros han explorado la perspectiva de los pacientes. ¿Por qué esta perspectiva es importante?

La enfermedad es antes que nada una experiencia individual, no pertenece por completo a los doctores o al estado y por lo tanto genera inquietudes, interpretaciones, significados y preguntas como: ¿Por qué yo? ¿Qué puedo hacer? ¿Cómo afecta mi vida? Hay algo intensamente subjetivo en cada enfermedad. Si entendemos como los pacientes percibieron la medicina y la enfermedad en el pasado podemos tener mayor simpatía y comprensión por el sufrimiento de las personas que actualmente están enfermas. Asimismo, la enfermedad y la muerte han sido dos de los mayores determinantes de la experiencia humana. El arte, la literatura, la moral, las relaciones sociales, las expectativas 
"El cuerpo no es sólo un ente biológico. Es semiótico. Es un sistema de signos y significados. Es un lenguaje en sí mismo". del futuro y la misma religión han evolucionado a la luz de la experiencia de la enfermedad y la muerte.

¿Qué influencias recibió al adoptar esta perspectiva de hacer una historia de la medicina desde abajo?

Creo que cuando estaba estudiando historia en la Universidad de Cambridge recibí una gran influencia del marxismo humanista relacionada en la historiografía inglesa con los trabajos de Edward P. Thompson, autor de The making of the working class. Esta corriente miraba con detalle cómo se habían construido las clases sociales y la experiencia misma de las clases trabajadoras y de los más pobres en este desarrollo. Esta perspectiva me impresionó mucho. Me pareció una manera de estar bajo la piel de la historia, una forma de entender cómo se enfrentaron en realidad los diferentes agentes en la historia, no según los dictados deterministas del marxismo científico, pero entendidos según un marxismo humanista. Llevé esta actitud a la historia de la medicina y me pregunté: ¿Cómo sería ver a la medicina, no desde arriba para abajo, pero desde la base hacia arriba? Creo que tenía sentido la pregunta porque no habría medicina si no existieran personas enfermas. La persona enferma es el comienzo de toda medicina.

\section{¿Tuvieron alguna influencia otras disciplinas como la antropología médica en esta perspectiva?}

Sí. En particular algunos antropólogos médicos como el norteamericano Arthur Kleinman, profesor en Harvard y autor de The illness narratives, suffering, healing and the buman condition, entre otros libros. Sin embargo, mi intención nunca fue tratar de agregar algo a lo que se hacía en la antropología médica o a la sociología médica, pero hacer algo diferente, paralelo, es decir en historia. Lo traté de hacer en parte porque los historiadores no le habían dado suficiente atención al problema de la enfermedad y la salud. Lo que quise hacer fue añadir algo a una nueva disciplina, si se quiere, que fue la historia social de la medicina. Otras perspectivas que creo que han sido muy influyentes en la historia social de la medicina, y que han influido no sólo mi propio trabajo, han sido el feminismo y la historia del cuerpo humano. El feminismo significó una manera de mirar lo familiar desde una perspectiva que no era común, desde una perspectiva no hegemónica. El cuerpo también ha sido estudiado, no sólo como un objeto de atención científica, sino como algo que tiene experiencias y significados para los individuos.

En sus trabajos también ha analizado el uso del cuerpo bumano en los discursos y en las caricaturas políticas.

El cuerpo no es sólo un ente biológico. Es semiótico. Es un sistema de signos y significados. Es un lenguaje en sí mismo. Tanto 
"Hay una ironía en la historia de la medicina. En los siglos anteriores al XX, cuando la medicina era menos científica, los doctores debían atender las necesidades de los pacientes que incluían la búsqueda de comprensión, empatía y esperanza”. en términos de sus apariencias como de sus movimientos, sus gestos y de sus comportamientos aprendidos. El cuerpo es además el mediador entre la intimidad de las personas y la sociedad en general. En los últimos años se ha transformado y enriquecido la sensibilidad de muchos investigadores e historiadores hacia el cuerpo humano.

También ha tratado de identificar lo específico de la medicina europea. ¿Existen algunas características propias de la medicina occidental?

A partir del renacimiento europeo del siglo XVI y de la revolución científica del siglo XVII la medicina occidental, a diferencia de otros sistemas médicos, se concentró en el cuerpo humano considerándolo el centro y muchas veces el origen de la enfermedad. A partir de entonces, la medicina occidental ha explorado, cada vez en mayor detalle, el cuerpo físico gracias a técnicas como la disección, el microscopio, el estudio de las células y la genética moderna. La diferencia con otros sistemas médicos como la medicina de la China o de la India es que estos últimos estuvieron mucho más preocupados por la relación del enfermo con la naturaleza y con el cosmos. No definieron la enfermedad según los cambios orgánicos internos en el cuerpo. Con relación a estos sistemas la medicina occidental ha tenido logros importantes pero ha vivido bajo el peligro de cierto reduccionismo y a veces ha prestado poca atención a los factores sociales que producen la enfermedad.

\section{Recientemente ha propuesto que la medicina occidental ba dado la} mejor explicación de la causa de las enfermedades pero no siempre ha ofrecido la mejor solución o medios de prevención. ¿Podría explicar esta idea?

Ese es parte del dilema de la ciencia y de la medicina modernas. Muchas veces los investigadores pueden explicar como funciona la naturaleza o la enfermedad pero no pueden aplicar inmediatamente este conocimiento. Ello se expresó en un nihilismo terapéutico durante el siglo XIX en el que los doctores sabían por qué la gente se moría pero no podían evitar estas muertes. También es importante decir que la práctica médica ha creado logros terapéuticos fundamentales que no siempre provienen de la investigación. Ha existido y existe una tensión entre la investigación y la práctica médicas.

Según sus estudios los médicos trascendian el rol de curary cumplian un rol familiary social, por ejemplo, acompañando al individuo doliente y desabuciado. ¿Quisiera decirnos algo al respecto?

Hay una ironía en la historia de la medicina. En los siglos anteriores al XX, cuando la medicina era menos científica, los doctores debían atender las necesidades de los pacientes que incluían la búsqueda de comprensión, empatía y esperanza. Es decir, los enfermos esperaban recibir más que un remedio. Cuando la medicina se hace científica 
adquiere cierta seguridad y descuida un poco escuchar a los pacientes para pasar a escuchar más a la enfermedad, a través del estetoscopio $\mathrm{y}$ los rayos $\mathrm{X}$, por ejemplo. Ello ha contribuido a que muchos enfermos sigan recurriendo a terapeutas no-biomédicos, especialmente en los países menos desarrollados, donde encuentran simpatía hacia ellos como personas.

¿Cuáles han sido los cambios más saltantes en los materiales que usa el historiador de la medicina interesado en la historia de lospacientes?

En los últimos años han habido grandes descubrimientos de nuevas fuentes históricas a las que en el pasado no se prestaba mucha atención. Así, por ejemplo, las fichas de hospital e historias clínicas, que antes no eran muy tomadas en cuenta por los investigadores, son cada vez más usadas. En mis trabajos de historia de la siquiatría descubrí muchos escritos autobiográficos de personas consideradas locas o enfermas mentales, algunas de las cuales estaban publicadas y otras no publicadas, y que no habían sido estudiadas previamente. Al leerlas me fue posible ir más allá del diagnóstico médico y descubrir la razón en la locura, la sistemática racionalización que hacían estos pacientes de sí mismos y de sus conductas.

Recientemente se ha criticado el énfasis exagerado porparte de algunos autores en los discursos médicos que son presentados como una forma de control social. Uno de sus libros examina con detalle estas críticas que han estado dirigidas a los trabajos de Michel Foucault.

Yo he sido muy influenciado por Foucault, pero muchas veces en una forma negativa. Es decir he tratado de ser activo en contra de algunas de sus ideas, de una forma que espero que sea creativa. Sin embargo, quiero dejar en claro que tengo un gran respeto por Foucault quien ha hecho contribuciones notables. Pero me parece que el énfasis de Foucault en la universalidad del discurso, o la presentación del discurso dictando la realidad y las personas limitándose a cumplir estos mandatos, limita la capacidad (agency) de los individuos a un nivel que me parece reduccionista y poco saludable. Por supuesto los defensores de Foucault dirán que esta es una interpretación cruda de Foucault, que él entendió las reacciones y la resistencia de las personas. Sin embargo, esa no es la manera en que yo leí a Foucault. Para mí es alguien para el cual la racionalidad y algunas ideas universales son los principales motores de la historia y simplemente no me gusta esta idea.

¿Es verdad que sus trabajos no siguen un modelo teórico sino que tienen cierto eclecticismo?

En realidad soy lo que a veces se denomina como un anticuado empirista anglo-sajón. Me gusta pretender que sigo lo que las evidencias históricas o los hechos me indican, considero que la 
"...soy lo que a veces se

denomina como un anticuado empirista anglosajón. Me gusta pretender que sigo lo que las evidencias históricas o los hechos me indican, considero que la narrativa es muy importante $y$ me gusta

examinar el caso individual y la experiencia individual..." narrativa es muy importante y me gusta examinar el caso individual y la experiencia individual, más que hacer un comentario general. No estoy muy interesado en grandes generalizaciones pero si me interesa comentar los grandes cambios que se producen en la historia, es decir los cambios que inciden en el largo plazo. Por eso escribí libros como The greatest benefit of mankind que empiezan en el pasado más remoto y llegan hasta el presente. Me fascina observar como la historia se hace sobre el pasado, como a veces rechaza el pasado o lo integra y como se llega a nuevas formas de pensar y de hacer las cosas. Así que el cambio histórico me parece cautivante, una perspectiva que creo que es diferente a la de Foucault, para el cual en realidad no hay cambios en la historia sino sólo diferentes discursos y distintas lecturas de los mismos. A mí me encanta estudiar como las cosas se transforman a sí mismas.

Uno de los aspectos más difíciles del trabajo como historiador es combinar adecuadamente la presentación del contexto histórico y la discusión de eventos particulares. ¿Ha seguido en sus trabajos alguna regla?

No creo haber seguido una metodología para ello. Tampoco me gustaría que a los historiadores se les dicte lo que deben de hacer según algún tipo de reglas o métodos. Me parece que cada historiador trae al material que analiza una serie de talentos, experiencias, prejuicios y expectativas que son únicas. Es precisamente de este diálogo entre el historiador y sus fuentes de donde surgen los buenos trabajos de historia. Al fin y al cabo si escribo un libro de historia siempre puede venir otro historiador usar las mismas fuentes y escribir un libro totalmente diferente. Es decir, no existe un trabajo de historia que sea definitivo.

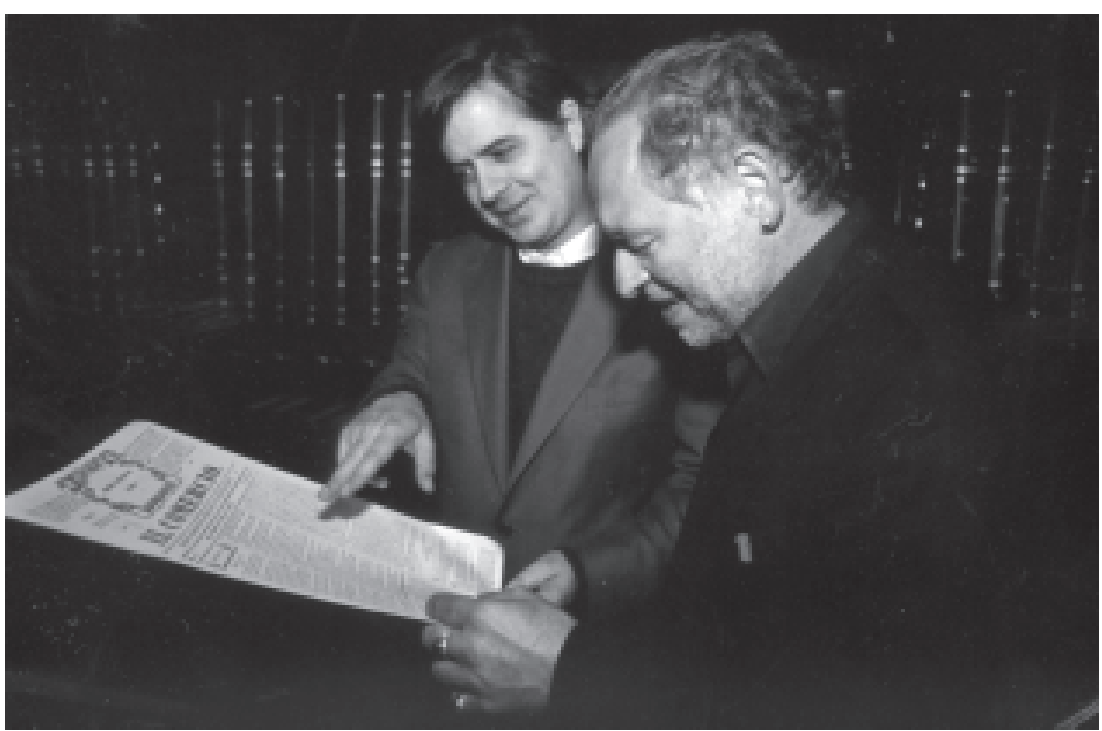

Marcos Cueto y Roy Porter 


“No busco el
estilo por sí
mismo. Me parece
que lo más
importante para
un académico es
comunicarse con
claridad con la
mayor cantidad de
personas que sea
posible”.

"No busco el estilo por sí mismo. Me parece que lo más importante para un académico es comunicarse con claridad con la personas que sea posible".
Sus trabajos le ponen gran atención al buen estilo y a la elegancia en la narración. Asimismo sus libros tratan temas diversos y están dirigidos a audiencias diferentes. ¿Por qué?

No busco el estilo por sí mismo. Me parece que lo más importante para un académico es comunicarse con claridad con la mayor cantidad de personas que sea posible. Sinceramente creo que mucha gente tiene mucho interés en conocer que pasó en la historia. Es verdad que con mis trabajos quiero llegar a una audiencia amplia y tratar de responder a la demanda del público por saber del pasado. Asimismo, yo personalmente estoy fascinado por una serie de temas sobre los que trato de escribir, lo cual en parte explica la diversidad de asuntos que he tratado. Creo que la historia general sin un entendimiento de la medicina se empobrece y que la historia de la medicina sin una comprensión de la sociedad, también se empobrece. Por ello, si puedo hacer las dos cosas y si puedo encontrar la manera en que se relacionan creo que podría hacer alguna contribución para ambos. También creo que una visión estrecha (narrowness) es un pecado que es frecuente entre los historiadores.

Es autor de una gran cantidad de publicaciones ¿Tiene un sistema para ser tan prolífico?

Es muy simple. Me levanto muy temprano en la mañana, alrededor de las seis de la mañana y empiezo a escribir, así no sienta ganas. Si lo hago sistemáticamente, para bien o para mal, siempre habré logrado escribir bastante. Si escribo hasta las nueve de la mañana por ejemplo - la hora en que empieza a llegar gente a la oficina - ya sé que he tenido algunas horas productivas, no importa cuantas interrupciones tenga en el resto del día. Tengo gran respeto por muchos de mis colegas que son perfeccionistas y que no publicarían nada hasta que todo lo que escriban esté en su lugar correcto. En cambio yo tengo un gran deseo de ensayar cosas nuevas y ver como resultan. Asimismo, trato de escribir y pensar al mismo tiempo lo cual creo que es esencial para que se me ocurran nuevas ideas. Siempre tengo terror de tener el bloqueo o la parálisis del escritor que no puede escribir o que está esperando la palabra perfecta.

En The greatest benefit of mankind describe la relación entre la medicina y la sociedad contemporáneas en la frase: "Haciéndolo mejor pero sintiéndose peor." ¿Qué quiere decir?

En los últimos cincuenta años muchas personas, especialmente en los países industrializados, se han sentido seguras ante la amenaza de infecciones. Esto ha cambiado la actitud del público hacia la profesión médica. En lugar de ver al doctor como el salvador de la muerte se le ve como el proveedor de una vida saludable y un cuerpo hermoso los cuales se consiguen con la cirugía cosmética, el Prozac o el Viagra. Al final acabamos en una espiral de insatisfacción 
porque las expectativas son infinitas. Muchas personas siempre querrán ser un poco más jóvenes, más sexy o vivir más tiempo. Uno de los problemas de la medicina actual es que las demandas que existen sobre ella no son realistas. La medicina está ahora contribuyendo a definir cuales son las expectativas razonables que pueden tenerse sobre la vida, la salud, la enfermedad y la muerte. ¿Por cuánto tiempo es realista esperar estar físicamente bien? ¿Cómo debemos afrontar el envejecimiento del cuerpo? Responder estas preguntas significará establecer más claramente el beneficio de la medicina para los seres humanos.

\section{¿Qué está escribiendo ahora?}

En este momento estoy escribiendo un libro sobre el cuerpo humano y el alma, viendo como la medicina y la religión interactúan. En este tema me parece que hay preguntas fundamentales: ¿Cómo se produce la transformación del mundo religioso al mundo médico o secularizado? ¿Cuál fue la manera en que lo trascendental o religioso se transformó en categorías médicas y científicas?

\section{BIBLIOGRAFÍA BÁSICA DE ROY PORTER}

En esta bibliografía sólo aparecen algunos de los libros de Porter en historia de la medicina.

1998

1997

1996

1994

1993

1993

1989

1989

1988

1988

1987

1987
Gout: The patrician malady. Editado con G. S. Rousseau. New Haven, Yale University Press.

The greatest benefit of mankind: a medical history of humanity. New York, W. W. Norton \& Company.

The Cambridge illustrated history of medicine. Cambridge, Cambridge University Press.

Reassessing Foucault: power, medicine and the body. Editado con Colin Jones. New York, Routledge.

Doctors, politics and society: historical essays. Editado con Dorothy Porter. Amsterdam, Rodopi.

Companion encyclopedia of the history of medicine. Editado con W. F. Bynum. London, Routledge.

Medicine and the five senses.

Editado con W. F. Bynum. Cambridge, Cambridge University Press.

Patient's progress: doctors and doctoring in eighteenth-century England. Editado con Dorothy Porter. Stanford, Stanford University Press.

Health for sale: quackery in England, 1660-1850.

Manchester, Manchester University Press.

In sickness and in bealth: The british experience, 1650-1850.

Editado con Dorothy Porter. New York, B. Blackwell.

A social history of madness: the world through the eyes of the insane. New York, Weidenfeld \& Nicolson.

Disease, medicine and society in England, 1550-1860.

Basingstoke, Macmillan Education.

Medical fringe and medical orthodoxy, 1750-1850

Editado con W. F. Bynum. London, Wolfeboro. 